\title{
Preoperative \\ Identification of Benefit \\ from Surgery for Malignant Pleural Mesothelioma
}

Isabelle Opitz, MD*, Katarzyna Furrer, MD

\section{KEYWORDS}

- Malignant pleural mesothelioma $\bullet$ Surgery $\bullet$ Survival $\bullet$ Prognostic factors

\section{KEY POINTS}

- Patients with malignant pleural mesothelioma have a poor prognosis.

- In current guidelines, surgery is the recommended option in selected patients with early-stage disease as part of a multimodal approach.

- Preoperative identification of factors associated with improved outcome is crucial for decisionmaking.

- The available literature for prognostic factors and scores for treatment allocation for surgery are presented.

\section{INTRODUCTION}

Malignant pleural mesothelioma (MPM) is a rare, aggressive, and devastating disease of the thoracic cavity associated with asbestos exposure. In recent years, MPM incidence has been rising and, although the use of asbestos has been prohibited in 55 countries, it is not expected to decrease until 2030. ${ }^{1,2}$ A report from the International Agency for Research on Cancer indicates that the disease burden is still substantial, with 30,443 cases of malignant mesothelioma and 25,576 deaths worldwide according to GLOBOCAN 2018 statistics. ${ }^{3,4}$ The treatment options are restricted by the poor prognosis and short life expectancy of 8 to 15 months ${ }^{5}$ from diagnosis. Surgery is part of the most effective multimodality treatment in prolonging survival and is a possible option for patients with a good prognosis in highvolume experienced centers. ${ }^{3}$

Selection criteria for surgery and identification of the appropriate candidate are crucial, and it is strongly recommended that a maximal surgical cytoreduction should be performed. ${ }^{6}$ Maximal surgical cytoreduction as a single-modality treatment is generally insufficient; additional antineoplastic treatment (chemotherapy and/or radiation therapy) should be administered. ${ }^{6}$ It is recommended that any treatment decision should be made with multidisciplinary input involving thoracic surgeons, pulmonologists, medical and radiation oncologists, radiologists, and pathologists to find the best therapeutic options for each individual patient. ${ }^{6}$

To provide recommendation for identification of benefit from surgery, many factors have to be taken into account: selection of candidates for surgery by clinical predicators and pathology with summary of the different predictor scores, as well as availability and access to different treatment approaches. This article summarizes these prognostic factors.

\section{CLINICAL AND RADIOLOGIC PREOPERATIVE PROGNOSTIC FACTORS}

Research focused on identification of clinical selection criteria for surgery in a multimodality

\footnotetext{
Department of Thoracic Surgery, University Hospital Zurich, Raemistrasse 100, Zurich 8091, Switzerland

* Corresponding author.

E-mail address: Isabelle.schmitt-opitz@usz.ch
} 
therapy approach was initiated in 1976, when Butchart and colleagues ${ }^{7}$ in Newcastle (UK) described the importance of precise patient selection for surgery. Butchart was one of the pioneers in mesothelioma surgery and concluded that death could have been prevented by better case selection, alteration in surgical technique, and better postoperative management. ${ }^{7}$

The unpredictable biological behavior of mesothelioma, the lack of correlation between clinical and pathologic staging, and patients' individual risk factors make the best treatment allocation difficult for an individual patient.

In general, specific considerations such as clinical factors (patients' demographics, performance status, specific prognostic markers in blood ${ }^{8-13}$ and pleural effusion, ${ }^{14}$ serologic markers of inflammation $^{15}$ and activated immune response, ${ }^{16,17}$ cardiac $^{18}$ and pulmonary assessments, ${ }^{19}$ radiologic staging, and tumor response to neoadjuvant chemotherapy) and pathologic factors (histology, genetic background, and molecular biomarkers), together with prognostic scores to select the patients for curative MPM surgery, are discussed in this article.

Historically, Butchart and colleagues ${ }^{7}$ divided their cohort of 29 patients in 1976 into "fit" and "unfit" for surgery and categorized them additionally into "above 60 years" or "below 60 years" of age. In general, age and, in early publications, white race were predictors for cancer-directed surgery, ${ }^{20}$ and advanced age was frequently mentioned as a significant factor associated with poor survival in previous reports. ${ }^{21-24}$ With changing demographics, age is currently a less stringent exclusion criterion for surgery; however, in the literature the age limit for favorable outcome varies from younger than 45 years $^{25}$ to younger than 70 years $^{6}$ (overall survival [OS] 19.8 vs 11.7 months; $P<.001$, multivariate analysis) ${ }^{26}$ as an independent prognostic factor, and surgery was associated with improved survival in patients aged $\geq 70$ years but not in those aged $\geq 80$ years in a Cox proportional hazards survival model published by Yang and colleagues. ${ }^{23}$ Consistent with these results, the Beijing group of Zhuo and colleagues $^{27}$ used the recent SEER (Surveillance, Epidemiology, and End Results) database to produce a nomogram showing that the mortality risk increases strongly with increasing age in patients older than 70 years. Thus, surgery on patients older than 70 years must be very carefully decided upon because it may not translate into a survival benefit. ${ }^{27}$ Another prognosticator of outcome and survival after surgery for MPM is gender. Female gender was associated with improved survival ${ }^{21,28}$ (OS 12.0 vs 9.9 months, $P<.001 ;^{29}$ and $22[95 \%$ confidence interval (Cl)] 18-30] vs 14 months $[95 \% \mathrm{Cl} 13-16]^{25}$ ). Male gender (hazard ratio [HR] $1.486,95 \% \mathrm{Cl} 1.241-1.77^{25}$ ) was independently associated with reduced OS (all $P<.05$ ). ${ }^{27}$ Circulating estrogen, present in young but not older women, and the expression of the estrogen receptor $\beta$, have been suggested to play a role in the survival difference between genders. ${ }^{30,31}$ In one of the first studies by Curran and colleagues ${ }^{10}$ the combination of poor performance status Eastern Cooperative Oncology Group (ECOG) status 1 or 2 , high white blood cell (WBC) count $\left(>8.3 \times 10^{\circ} / \mathrm{L}\right)$, and male gender were in general associated with poor prognosis, ${ }^{32,33}$ whereas ECOG status 0 (27.4 vs 9.7 months; $P=.015$ ) was a preoperative factor predicting benefit for surgery and survival. ${ }^{29}$ Analyses of large datasets defined negative prognostic factors such as poor performance status (PS), low hemoglobin $(\mathrm{Hb})$ count, male gender, high platelet count, high lactate dehydrogenase (LDH) level, and high WBC count. ${ }^{12}$ Neutrophil-to-lymphocyte ratio (NLR) less than 5 (11.9 vs 7.5 months; $P<.001$ ), platelet count less than $400 \mathrm{G} / \mathrm{L}(11.5 \mathrm{vs}$ 7.2 months; $P<.001)$ and normal $\mathrm{Hb}(16.4$ vs 8.8 months; $P<.001)$ are factors predicting benefit for MPM surgery and long-term survival. ${ }^{29,34}$ C-reactive protein (CRP) is a typical inflammation-related independent prognostic biomarker (HR 2.07, 95\% Cl 1.23-3.46; $P=.01$ ), and patients with increased levels in blood had shorter OS in comparison with normal CRP (CRP $\geq 1 \mathrm{mg} / \mathrm{dL}$ : HR 2.81, 95\% Cl 1.82-4.33; $P<.001) .{ }^{16}$ Furthermore, increased CRP in pleural effusion $^{35}$ was associated with worse outcome (CRP $\geq 3.8 \mathrm{mg} / \mathrm{dL}:$ HR 2.288, Cl 1.505-3.478; $P<.001){ }^{36}$

Other hematologic markers associated with diagnosis and prognosis (discussed in detail in Harvey I. Pass and colleagues' article, "Mesothelioma Biomarkers: Discovery in Search of Validation," in this issue) include soluble mesothelin-related proteins, osteopontin, Fibulin3, high-mobility group box 1 (HMGB1), lymphocyte-to-monocyte ratio (LMR), NLR, and platelet-to-lymphocyte ratio (PLR). ${ }^{37,38}$ HMGB1, a damage-associated molecular pattern protein released by necrotic cells, has diagnostic and prognostic value, ${ }^{39,40}$ with an inverse association between HMGB1 serum levels and survival at a cutoff value of $9 \mathrm{ng} / \mathrm{mL}$. Lastly, the most promising hematologic marker is the peripheral blood marker LMR, given its proven correlation with survival. Yamagishi and colleagues ${ }^{41}$ showed that patients with LMR serum level greater than 2.74 had longer OS of 14 months in comparison with 5 months at a lower level. 
Current guidelines of the British Thoracic Society (BTS) recommend the use of specific factors to determine prognosis at baseline and timing of treatment. ${ }^{42}$ These comprise demographic factors (age, gender, race), disease features (histologic subtype and grade, site of disease, disease stage using various staging systems), ECOG PS or Karnofski performance score, symptoms (chest pain, weight loss), total WBC count, platelet count, NLR, PLR, CRP level, and blood test markers such as $\mathrm{Hb}$ level, $\mathrm{Hb}$ difference from a population ideal value (160 $\mathrm{g} / \mathrm{L}$ in men, $140 \mathrm{~g} / \mathrm{L}$ in women), and serum albumin (all grade D). ${ }^{42}$

Concerning the application of the of the non-tissue-based biomarkers for diagnosis, predicting outcome, or monitoring tumor response, the current American Society of Clinical Oncology (ASCO) guidelines state that these markers are under evaluation and at this time do not have the sensitivity or specificity to predict outcome or monitor tumor response, and are therefore not recommended (Type of recommendation: evidence based; Evidence quality: intermediate; Strength of recommendation: moderate). ${ }^{6}$

The selection process based on radiologic features to decide which patients are eligible for surgery is challenging and requires expertise and interdisciplinary collaboration. According to current ASCO guidelines, patients with transdiaphragmatic disease and multifocal chest wall invasion (ie, all features that exclude patients from macroscopic complete resection [MCR]) should undergo neoadjuvant treatment before consideration of maximal surgical cytoreduction. ${ }^{6}$ These staging questions should be addressed by the following investigations: computed tomography (CT) scan with intravenous (IV) contrast (chest and upper abdomen) and $\left[{ }^{18} \mathrm{~F}\right]$ fluorodeoxyflucose PET/CT $\left({ }^{18} \mathrm{~F}-\mathrm{FDG}\right.$ PET/CT) scan (Type of recommendation: evidence based; Evidence quality: intermediate; Strength of recommendation: strong) or MRI, particularly with IV contrast (Type of recommendation: evidence based; Evidence quality: intermediate; Strength of recommendation: moderate), as recommended by ASCO guidelines. ${ }^{6}$

In accordance with BTS guidelines, concerning imaging modalities for diagnosing and staging, CT scan of thorax with IV contrast is recommended for the initial imaging modality as well as PET/CT to exclude distant metastases-but not for aiding diagnosis in patients who have had prior talc pleurodesis and with caution in populations with a high prevalence of tuberculosis - in addition to MRI for patients in whom differentiating $T$ stage will change management (all grade D). ${ }^{42}$ In general, the radiologic staging often has a poor correlation with pathologic staging. ${ }^{43}$ Additional radiologic assessments with functional imaging techniques using MRI, such as diffusionweighted imaging (DWI) and dynamic contrastenhanced (DCE) MRI with ${ }^{18} \mathrm{~F}-\mathrm{FDG}$ PET/CT, can rule out chest wall infiltration, transdiaphragmatic infiltration, nodal involvement, or occult metastasis at the same time. ${ }^{44,45}$

Although staging algorithms for MPM are discussed in detail in R. Taylor Ripley's article, "Extended Pleurectomy and Decortication for Malignant Pleural Mesothelioma," in this issue, a word about exclusion of patients from surgery based on clinical stage is timely. Overall, the decision to undergo surgery should still be made on an individual basis.

Traditionally, TNM stage is the classic prognosticator for cancer treatment. Both parameters $-T$ stage and $\mathrm{N}$ stage-combined with epithelial histology, female gender, and adjuvant therapy are the traditionally identified prognostic factors in patients with $\mathrm{MPM}^{46}$; however, nowadays the discrimination between T2 and T3 (infiltration of chest wall) is almost impossible. Moreover, there are no precise selection criteria based on $\mathrm{T}$ factors alone. ${ }^{47-49}$ The current American Joint Committee on Cancer/Union for International Cancer Control classification ${ }^{50}$ mentions difficulties in applying it to clinical staging with respect to both $\mathrm{T}$ and $\mathrm{N}$ parameters, resulting in imprecise predicted prognosis; moreover, as strongly recommended in ASCO guidelines, clinicians should recognize that in patients with clinical stage $1 /$ II disease, upstaging may occur at surgery. ${ }^{6}$ In the last proposals for revision of the $T$ descriptors, survival correlated with pleural thickness. Pleural thickness showed an increase at higher $\mathrm{T}$ stages and was significantly associated with node positivity and overall stage. Based on these findings and data from the seventh edition of $T$ categories and overall stage, survival showed a median of 23.4 months for the lowest tumor thickness $(<16.0 \mathrm{~mm})$ vs 13.2 months for the highest tumor thickness $(>50.0 \mathrm{~mm}) .{ }^{51}$ Nevertheless, further investigations as to whether tumor thickness should be included in future staging systems are necessary. Tumor stages I to III are included if deemed technically resectable, with most of the patients treated within clinical trial protocols. Decisions leading to surgery are made individually, and even localized chest wall infiltration is accepted if chest wall resection seems feasible and reasonable at only one level.

In this regard, another important prognosticator is the status of mediastinal lymph node involvement ( $\mathrm{N}$ factor), which is a poor prognostic factor for MPM as mentioned in several studies 2 decades ago. ${ }^{46,52,53}$ Mediastinal staging is 
performed with PET/CT, EBUS/FNA (endobrachial ultrasound elastography/fine-needle aspiration), or mediastinoscopy to exclude N3 disease. ${ }^{54} \mathrm{~Pa}$ tients with histologically confirmed contralateral mediastinal or supraclavicular lymph node involvement should undergo neoadjuvant treatment before consideration of maximal surgical cytoreduction according to recent ASCO guidelines. ${ }^{6}$ The proposal for current eighth edition of the International Association for the Study of Lung Cancer Mesothelioma Staging Project showed that for clinically staged tumors there was no difference between cN0, cN1, or cN2 (cN1 vs cNO: HR 1.06, $P=.77$ and $\mathrm{cN} 2$ vs $\mathrm{cN} 1$ : HR 1.04, $P=.85)$, and patients with pN1 or pN2 tumors had shorter survival than those with $\mathrm{pNO}$ tumors (HR 1.51, $P<.0001$ ), but no survival difference was observed between those with pN1 and pN2 tumors (HR 0.99, $P=.99$ ). Patients with concurrent $\mathrm{pN} 1 / \mathrm{pN} 2$ nodal involvement had poorer survival than those with pN2 tumors alone (HR 1.60, $P=.007)$ or pN0 tumors (HR 1.62, $P<.0001){ }^{55}$ Occult nodal disease detected during resection for CNO MPM correlate with higher hazard of mortality $(P=.005)^{56}$ and poorer prognosis with similar survival as $\mathrm{cN}+$ cases. These data underline the importance of routine preoperative pathologic nodal assessment for potentially resectable MPM and that the number of involved lymph nodes (rather than current location-based classification) is associated with OS and may provide more robust prognostic stratification for future TNM staging. ${ }^{56}$

In conclusion, this means that nodal involvement seems to be of prognostic importance, although the location in the mediastinum needs to be interpreted differently from lung cancer. Therefore, the $\mathrm{N}$-descriptor was revised for the eighth TNM classification to more MPM-specific $\mathrm{N}$-categories in comparison with the seventh edition, where the median survival for cNO patients was 19 months vs 17.6 and 16.2 months for cN1 and cN2 patients, respectively, without statistical significance. ${ }^{55}$ Surgery alone for patients with positive ipsilateral mediastinal lymph nodes is not appropriate, and a multimodality approach, particularly as part of a clinical trial, should be considered. ${ }^{6}$

Selecting MPM patients for surgery based on response to chemotherapy or other treatments as a marker for biological behaviors is another opportunity. However, radiologic assessment of tumor response to treatment using standard RECIST (Response Evaluation Criteria in Solid Tumors) criteria that require bidimensional measurements is not practical and often difficult in monitoring of mesothelioma, ${ }^{57}$ because the morphology and growth pattern of mesothelioma differs substantively from that of other solid tumors. ${ }^{58}$ Based on restaging imaging by contrastenhanced CT or ${ }^{18}$ F-FDG PET/CT imaging after induction chemotherapy and assessment of modified RECIST (mRECIST) criteria, ${ }^{59}$ patients are classified as progressive disease, stable disease, or partial response, and whether they are potentially resectable or not. This method requires acquisition of up to 6 measurements of tumor thickness, each at least $1 \mathrm{~cm}$ in extent, perpendicular to the chest wall or mediastinum ${ }^{59,60}$ with no more than 2 measurement sites on each of 3 separate CT sections separated axially by at least $1 \mathrm{~cm},{ }^{59}$ involving a multistage process with variability between observers at each of these steps. ${ }^{61}$ Consequently, this should be performed by a radiologist familiar with mRECIST for MPM. ${ }^{62}$ Complete or partial radiologic response is associated with improved median survival of 26.0 vs 13.9 months for patients with stable disease or progressive disease $(P=.05) .{ }^{63}$ However, progressive disease alone is not an exclusion criterion per se for surgery as long as MCR is still feasible (Fig. 1 and Opitz and colleagues, unpublished data).

For a more accurate evaluation of resectability in terms of tumor load, tumor volume measured before and after induction treatment is an attractive parameter. Conventional quantitative measurement of the tumor volume (Fig. 2) ${ }^{47}$ and newly deep convolutional neural network (CNN)automated volumetric segmentation of MPM tumor on CT scans ${ }^{64}$ has been a field of interest for prognostic evaluation of tumor staging ${ }^{65}$ and response with reduction in tumor volume on $\mathrm{CT}^{66}$ or $\mathrm{MRI}^{67}$ after neoadjuvant treatment, ${ }^{47,68,69}$ as well as prediction of survival. ${ }^{66}$ Furthermore, already observed increasing tumor volume and decreasing lung volume during neoadjuvant chemotherapy are both significantly and independently associated with poor prognosis. ${ }^{70}$

As described first in 1998 by Pass and colleagues, ${ }^{71}$ preoperatively assessed tumor volume can predict OS and progression-free survival (PFS) as well as postoperative stage. Large volumes are associated with nodal spread as well as postresection residual tumor burden and may predict the outcome. ${ }^{47,65,71,72}$ In another study, Gill and colleagues ${ }^{73}$ showed poorer OS if the tumor volume was greater than $500 \mathrm{~cm}^{3}$, confirmed by Rusch and colleagues, ${ }^{65}$ who reported that tumor volumes of $91.2,245.3$, and $511.3 \mathrm{~cm}^{3}$ were associated with a median OS of 37,18 , and 8 months, respectively.

Radiomic biomarkers had a stronger prognostic value compared with tumor volume alone. The 


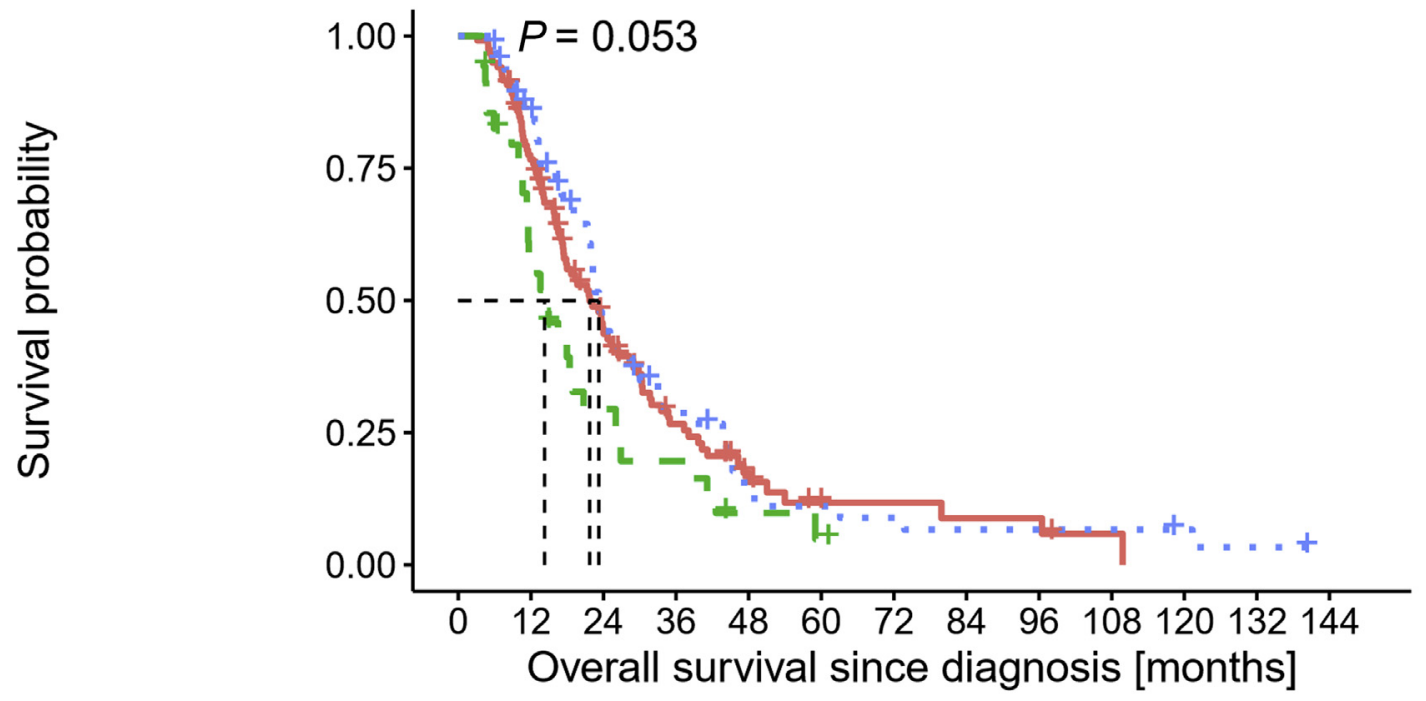

Number at risk

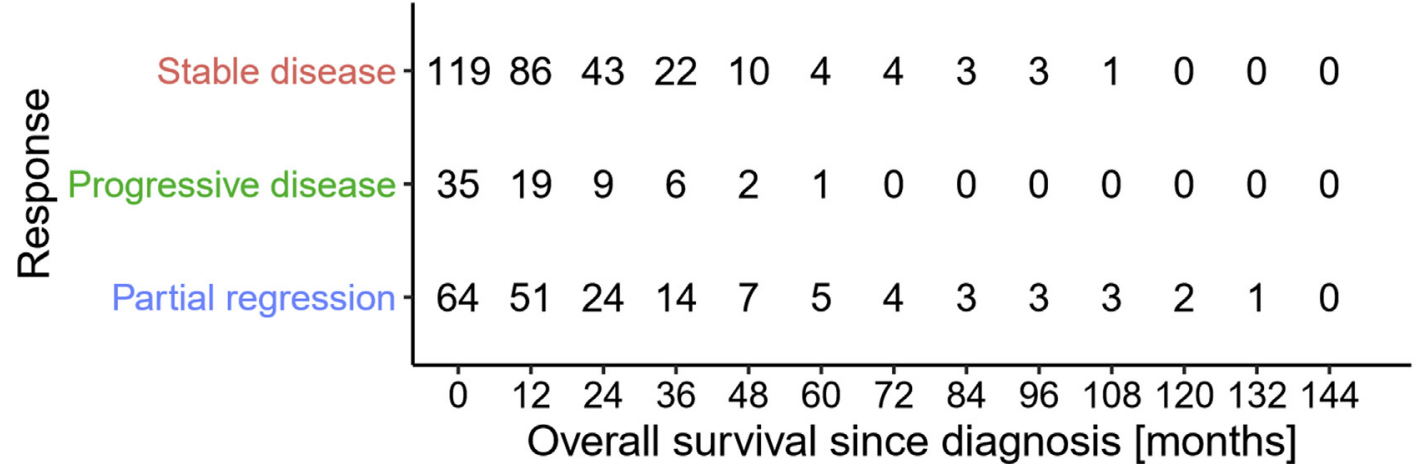

Fig. 1. Intention to treat (ITT) by response defined according to mRECIST criteria by independent observers (T.F., C.Z., D.-L.N.) ${ }^{59}$ in $\mathrm{N}=218$ patients before and after induction as was the tumor volume, which was assessed by the help of semiautomated dedicated software as described previously. ${ }^{47}$ Partial regression does not seem more beneficial compared with stable disease when considering survival times (HR, 0.9067194; Cl, [0.6404001, 1.2837914]; $P=.58$ ). However, a progressive disease response is associated with a significantly higher risk of dying compared with a stable disease response (HR, 1.5496089; $\mathrm{Cl},[1.0230936,2.347085] ; P=.04)$.
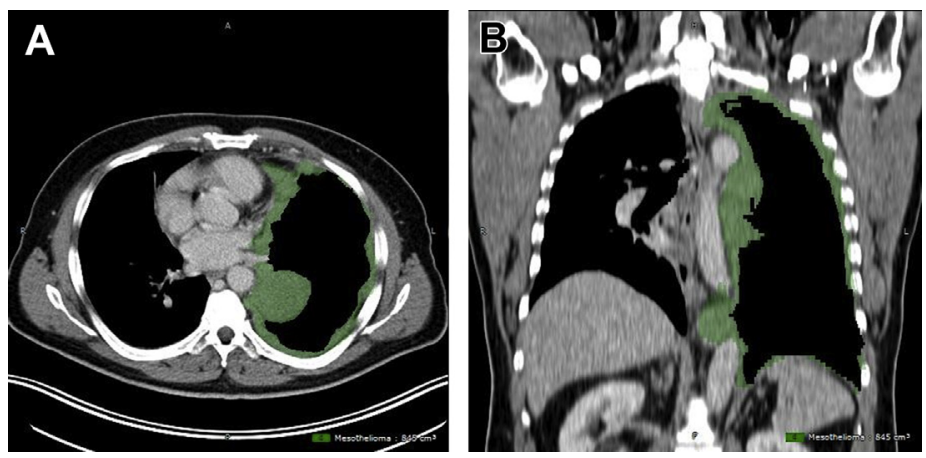

Fig. 2. Volumetry of malignant pleural mesothelioma. $(A, B)$ The marked tumor (green) on a single CT slice. ${ }^{47}$ 
authors evaluated radiomic features (shape, intensity, texture [relation of an individual pixel to its neighborhood], and wavelet decomposition), a promising methodology for quantitative analysis and description of radiologic images using advanced mathematics and statistics in CT images, and a prognostic model for OS in MPM patients was developed based on these CT image characteristics. ${ }^{74}$ Two wavelet features were prognostic for OS in multivariable Cox regression (concordance index: $0.74 ; P=.002$ ). Both features separated the patients into 2 groups with a significantly different OS $(P=.0006)$ and a significantly different PFS $(P=.003) .{ }^{69}$

MRI offers higher contrast resolution than CT and is therefore potentially more appropriate for volumetric analyses. ${ }^{75}$ Tumor volumes $(\geq 300 \mathrm{~mL})$ had significantly poorer median OS (multivariable Cox proportional hazards model, HR 2.114 [1.046-4.270], $P=.037) .{ }^{69}$ However, the observed difference in median OS with tumor volume increased when analysis was restricted to patients with epithelioid histology and further increased when epithelioid cases with nodal or metastatic disease were excluded. ${ }^{69}$

Other functional imaging techniques using DWI and DCE MRI have the potential to act as a quantitative method of assessing tumor response to treatment. ${ }^{57}$ These techniques are reflective of the underlying tumor pathophysiology, such as tissue cellularity and microvessel density. ${ }^{57}$ In the analysis of preoperative prediction of unresectability in MPM, Burt and colleagues ${ }^{76}$ proposed a novel metric of thoracic cage volume, calculated by preoperative chest CT scan, and determined associations between preoperative variables and diffuse chest wall invasion (DCWI) and contraction of thoracic cage volume in patients scheduled for MCR. Decreased ipsilateral thoracic cage volume demonstrated the strongest association with unresectability by DCWI $(P=.009)$, with greater than $5 \%$ decrease representing the optimal cutoff $(P=.014$; area under the curve, 0.67). Preoperative identification of DCWI can avoid unnecessary thoracotomy and accelerate initiation of nonsurgical therapy. ${ }^{76}$ Other variables associated with DCWI included chest pain requiring opioids $(P=.028)$, pleurodesis $(P=.036)$, decreased forced vital capacity (FVC) $(P=.023)$, decreased ipsilateral lung perfusion $(P=.007)$, and chest wall invasion $(P=.035){ }^{76}$

Another potential selection tool for surgery or supportive factor in the decision-making process is the prognostic significance of maximum standardized uptake value $\left(\mathrm{SUV}_{\max }\right)$ as assessed by ${ }^{18} \mathrm{~F}-\mathrm{FDG}$ PET/CT, which measures tumor activity and functional tumor volume to indicate patient prognosis ${ }^{77}$ and is the focus of continued research and clinical investigation. ${ }^{69}$ However, PET should be interpreted with caution in patients after talc pleurodesis. ${ }^{6}$ Recently, Lim and colleagues ${ }^{78}$ proposed SUV $\mathrm{Vax}_{\text {max }}$ as an independent prognostic factor in all patients $(P=.003)$, especially those with the epithelioid subtype associated with OS $(P=.012)$, but not in those with a nonepithelioid subtype.

Finally, an important factor is the patients' individual expectation of postoperative quality of life (QoL). Despite not unsubstantially reduced overall QoL regarding ability to perform everyday activities that reflect physical, psychological, and social well-being, and patient satisfaction with levels of functioning and control of the disease after surgery, some of these studies demonstrate a tendency for better QoL (physical function, social function, and global health were better at followup) after lung-sparing surgery ${ }^{79}$ after 6 and 12 months, ${ }^{80}$ whereas other indicators such as pain and cough were similar. ${ }^{79}$ This might be related to the fact that with decortication of entrapped lungs, lung function and, therefore, QoL can improve. Predicted postoperative $\mathrm{FEV}_{1}$ (forced expiratory volume in $1 \mathrm{~s}$ ) and FVC were reported in one study only and were higher at follow-up for pleurectomy/decortication (P/D) compared with extrapleural pneumonectomy (EPP). ${ }^{81}$

\section{Pathologic Subtypes, and Genetic and Molecular Factors}

Benefit from surgery and survival for MPM is significantly affected by tumor histology. The histologic subtypes of MPM with epithelioid, sarcomatoid, and biphasic type have clear prognostic significance, ${ }^{6}$ which might help in selecting patients for intensive multimodal treatment approaches and in identifying whether patients are eligible for surgery. The biphasic subtype showed poor prognosis compared with the epithelioid subtype, whereas prognosis of the sarcomatoid subtype was worst in a recently published analysis of the SEER database ${ }^{27}$ and consistent with previous studies and guidelines. ${ }^{6,8,10,29,52,53,82-84}$ Meyerhoff and colleagues ${ }^{24}$ reported that most common epithelioid histology is related to better survival compared with biphasic or sarcomatoid histology (19 vs 12 vs 4 months in the sarcomatoid group $[P<.01])$ and in multivariate analysis, surgery was associated with improved survival in the epithelioid group (HR 0.72, $P<.01)$ but not in biphasic (HR 0.73, $P=.19$ ) and sarcomatoid (HR $0.79, P=.18$ ) groups regardless of type of surgical resection. 
According to the World Health Organization 2015 classification ${ }^{83,85}$ of epithelioid MPM, histologic subtypes of predominantly microcystic/tubulopapillary pattern were associated with longer OS than the solid/trabecular subtype (732 vs 397 days, $P=.0013)$, whereas the pleomorphic subtype had the shortest OS (173 days). ${ }^{86}$ The solid/trabecular variants showed a significant association with a high nuclear grade and mitosisnecrosis score as an independent prognostic factor. ${ }^{86}$ In a group of patients who received multimodal treatment, those with tubulopapillary/ microcystic pattern MPMs showed a tendency toward better OS than those with solid/trabecular pattern tumors (HR 2.29, 95\% Cl 0.95-5.12; $P=.066) .{ }^{86}$

Another current subject of further investigation is grading, whereby well or moderately differentiated subtypes resulted in better prognoses than poorly differentiated or undifferentiated subtypes, ${ }^{27}$ suggesting that nuclear atypia and mitotic count are independent preoperative prognostic markers for MPM. ${ }^{83}$

In conclusion, according to ASCO guidelines surgery is not recommended in patients with sarcomatoid $\mathrm{MPM}^{6}$; however, in carefully selected cases biphasic or sarcomatoid subtype with negative mediastinal lymph node status is not obliged to be excluded from surgery because this can be the only option for these patients, given their higher incidence of chemoresistance. ${ }^{52,87,88}$ Therefore, the decision for or against surgery should not be based on a single factor such as histotype but rather a combination of various factors, given that there are certain subtypes of epithelioid MPM - biphasic or sarcomatoid histotypes - with proven long-term survival. ${ }^{89,90}$

Genetic predisposition, as explored in preclinical studies, is an important predictor of disease progression and survival (discussed in detail in Benjamin Wadowski and colleagues' article, “The Molecular Basis of Malignant Pleural Mesothelioma," in this issue). To date, it has not been included in the decision-making process for or against surgery. In brief, genetic prognosticators include chromosome alterations of cyclindependent kinase inhibitor 2A (CDKN2A) locus (9p21.3), homozygous p16 deletions (especially for sarcomatous type), and BRCA1-associated protein 1 (BAP1) mutations, all of which are associated with poor prognosis. ${ }^{91-93}$

In the following a few molecular prognostic factors, being a focus of the authors' research area, are briefly discussed, though not in depth because this is the topic of Harvey I. Pass and colleagues' article, "Mesothelioma Biomarkers: Discovery in Search of Validation," in this issue. However, recommendations regarding the use of biomarkers from current guidelines ${ }^{42}$ do not support biomarkers in isolation as a diagnostic (Grade B) or screening test (Grade $C$ ) or to predict treatment response or survival (Grade B) in MPM.

MicroRNAs (miRNAs) are small noncoding RNA molecules that regulate gene expression. ${ }^{94,95}$ Kirschner and colleagues ${ }^{96}$ established the miRScore, consisting of 6 miRNAs (miR-21-5p, -23$3 p,-30 e-5 p, 221-3 p,-222-3 p$ ) for prediction of longer survival in positive patients.

MPM is characterized by complex chromosomal aberrations, including chromosome 10 losses and the tumor-suppressor gene phosphatase and tensin homolog deleted from chromosome 10 (PTEN) located on chromosome 10q23. The authors' group showed that median survival time was significantly longer in patients with PTEN expression (15.5 months: $95 \% \mathrm{Cl} 3.8,27.2$ vs 9.7 months: $95 \% \mathrm{Cl} 7.9,11.7)$ independent of histologic subtype $(P=.7){ }^{97,98}$ Furthermore, Schramm and colleagues $^{99}$ demonstrated that low cytoplasmic periostin and high cytoplasmic PTEN are independent prognosticators of better OS. ${ }^{99}$ In addition, the authors observed that a decrease in PTEN and an increase in p-mTOR (pathologic mammalian target of rapamycin) expression during induction chemotherapy were associated with shorter OS. ${ }^{98}$ These investigations were recently confirmed by Kuroda and colleagues, ${ }^{100}$ who moreover proposed that molecular-targeted treatment involving the mTOR signaling pathway might be used during multimodal therapy for MPM.

Another prognosticator is cytokine migration inhibitory factor (MIF) and its receptor CD74 together with calretinin in tissue microarray, which correlates with OS. CD74 $(P<.001)$ but not MIF overexpression $(P=.231)$ is an independent prognostic factor for prolonged OS. ${ }^{101}$ Interestingly these positive results for CD74 were consistent with a previously published report that high expression of tumoral PTEN is an independent prognostic factor for prolonged OS in mesothelioma patients. ${ }^{97}$ High expression of tumor cell calretinin correlated with the epithelioid histotype predicted longer OS $(P<.001) .{ }^{101}$ Thies and colleagues $^{102}$ correlated the neural crest stem cell marker nestin and the epithelial-mesenchymal transition marker periostin with histology and found that in platinum/pemetrexed-treated patients, nestin was higher in biphasic MPM compared with epithelioid MPM, Regarding expression of nestin in chemo-naïve biopsies (OS: 22 vs 17 months) and chemo-treated surgical specimens (OS: 18 vs 12 months), both nonepithelioid histology and high periostin level in biopsies (OS: 23 vs 15 months) were associated with poor 
prognosis. In the multivariate survival analysis, any nestin expression in chemo-naïve biopsies proved to be an independent prognosticator against histology. ${ }^{102}$ Furthermore, Meerang and colleagues ${ }^{103}$ discovered that low Merlin expression and high Survivin expression are also associated with a poorer prognosis. These investigators showed that cell proliferation marker $\mathrm{Ki}-67$ and a high nuclear Survivin-labeling index in prechemotherapy and postchemotherapy tissues were associated with shorter freedom from recurrence. $^{98,103}$ Programmed death-ligand 1 (PD-L1) expression in tumor tissue was associated with a lower median survival of 6 vs 15.5 months compared with negative PD-L1, and positive PDL1 expression $(\geq 1 \%)$ was independently correlated with poor prognosis (HR 2.02, 95\% Cl, 1.005-4.057, $P=.0484) .{ }^{104}$ Patients with PD-L1positive tumors had shorter OS than patients with negative PD-L1 (HR 1.581, Cl 1.043-2.396, $P=.031) .{ }^{36}$

In summary, although all of these markers showed promising results, most have yet to be independently and prospectively validated, and for some of them controversial results from different studies concerning their prognostic impact need to be addressed. ${ }^{38}$ So far, all these markers and their potential prognostic value have had no impact on patient selection in predicting outcome or monitoring tumor response, ${ }^{6}$ but may be included in selection algorithms.

\section{PROGNOSTIC SCORES}

Because the use of a single factor to predict prognosis is not justified, with rather the synthesis of several factors leading to the decision for a curative operation, prognostic scores are of interest. Combining groups of prognostic variables originating from cohorts of patients and subsequent validation in different test cohorts ${ }^{42}$ seems to be attractive for the identification of candidates for surgery. The European Organization for Research and Treatment of Cancer (EORTC) and the Cancer and Leukemia Group B (CALGB) developed a prognostic score and prognostic groups for better identification of patients receiving different chemotherapy regimens by analyzing the patients " pretreatment characteristics. ${ }^{10,11}$

The EORTC prognostic score ${ }^{10}$ includes the ECOG PS, histologic subtype, gender, certainty of diagnosis (definitive vs possible), and defined good prognosis group (ECOG 0, epithelioid histology, female, definitive diagnosis, and WBC $<8.3 \times 10^{9} / \mathrm{L}$ ) and poor prognosis group (ECOG 1, nonepithelioid histology, male, possible diagnosis, and WBC > $8.3 \times 10^{9} / \mathrm{L}$ ). The CALGB prognostic score is derived from 6 prognostic subgroups. Here poor ECOG PS, chest pain, dyspnea, platelet count greater than $400 \mathrm{G} / \mathrm{L}$, weight loss, $\mathrm{LDH}$ level greater than $500 \mathrm{IU} / \mathrm{L}$, pleural involvement, low $\mathrm{Hb}$ level, high WBC count, and age greater than 75 years predicted poor survival. Pleural involvement, LDH greater than $500 \mathrm{IU} / \mathrm{L}$, poor PS, chest pain, PLT greater than 400,000 / $\mu \mathrm{L}$, nonepithelial histology, and age greater than 75 years predicted poor survival in multivariate analysis. ${ }^{11}$ The subgroup with the best survival included patients with PS 0 and age less than 49 years, and patients with PS 0 , age 49 years or older, and hemoglobin $\geqq 14.6 \mathrm{~g} / \mathrm{L}$. The worst survival occurred for patients with PS $1 / 2$ and WBC $\geqq 15.6 / \mu \mathrm{L} .{ }^{11}$

Edwards and colleagues ${ }^{12}$ validated the effectiveness of the EORTC and CALGB scores as early as 20 years ago. Patients were stratified into lowrisk and high-risk groups and correlated with the EORTC series with a median survival of 9.4 vs 10.8 months (low-risk group) and 3.8 vs 5.5 months (high-risk group), respectively. ${ }^{12}$. The EORTC score was recently evaluated by the Italian group of Sandri. ${ }^{105}$ Multivariable analysis confirmed an independent prognostic value of EORTC score (HR 2.86, $P<.001)$ as a reliable and valid instrument that may be implemented in daily practice. ${ }^{105}$

Furthermore, an Italian group explored predictors of long-term survival and defined a prognostic score in a multicenter analysis. ${ }^{106}$ On multivariate analysis, younger age $(\mathrm{OR} 0.51,95 \% \mathrm{Cl} 0.31-$ 0.82 ), epithelioid histology (OR 7.07, 95\% Cl 1.56-31.93), no history of asbestos exposure (OR $3.13,95 \% \mathrm{Cl} 1.13-8.66)$, and the ratio between metastatic and resected lymph nodes less than 22\% (OR 4.12, 95\% Cl 1.68-10.12) were independent predictors of long-term survival. Long-term survival was defined as survival longer than 2 times the median OS (18 months) and stratified patients into 2 groups: 36 months (long-term survival group) and those surviving between 4 and 35 months (short-term survival group). The investigators created a scoring system (1-12 points) identifying patients for long-term survival with a score greater than 6 and predicting favorable overall, cancer-specific, and disease-free survival $(P<.0001) .{ }^{106}$

Pass, Rusch, and colleagues ${ }^{107,108}$ identified prognostically important and independent covariates for prediction of survival including stage, age, gender, histology (epithelioid vs nonepithelioid), and the type of surgical procedure (palliative vs curative), defined as "CORE" values, and analyzed their impact on OS: adjuvant therapy (yes: OS 18 months vs no: OS 10 months), smoking history (no: OS 16 months vs yes: OS 
15 months), history of asbestos exposure (no: OS 17 months vs yes: OS 15 months), history of weight loss, defined as greater than $5 \%$ (OS 11 months) vs less than 5\% (OS 17 months) in the previous 6 months, ECOG PS 0 (OS 22 months) and 1 (OS 16 months), chest pain (no: OS 19 months vs yes: OS 14 months); and dyspnea (no: OS 15 months vs yes: OS 17 months), Hb level (<14.6; OS 16 months vs $>14.6$ OS 20 months), platelet count $(<400$; OS 19 months vs $>400$ OS 12 months), WBC count (<15.5; OS 16 months vs $>15.5$ OS 8 months), and LDH level before surgery. In total, they defined 3 prognostic models with these covariates. Models 1 and 2 included the CORE variables. Model 1 additionally included adjuvant treatment, WBC, and platelet count, and model 2 consisted of the same covariates as model 1 but without a surgical staging, with $\mathrm{Hb}$ added as additional parameter. Model 3 showed only parameters available before surgery (histology, gender, age, WBC, $\mathrm{Hb}$, and platelets) and therefore represented the potential surgery patient. The models per se were not analyzed according to their prognostic value; only their covariates were individually indicated as having prognostic significance and HR. ${ }^{107,108}$

The modified Glasgow Prognostic Score (mGPS) categorizes patients with cancer according to CRP and serum albumin. ${ }^{15}$ This was found to be an independent predictor of OS in MPM (HR 2.6, 95\% Cl 1.6-4.2, $P<.001)^{15}$ and has not been further prospectively validated. Both mGPS and NLR were independent predictors of OS (HR 2.6 and 2.0 , respectively). ${ }^{15}$
The LENT prognostic score was developed by Clive and colleagues ${ }^{109}$ for predicting survival in patients presenting with malignant pleural effusion using LDH (>1500) IU/L, ECOG PS, NLR, and tumor type. Analysis of the area under the receiveroperating characteristic (ROC) curve revealed the LENT score to be superior at predicting survival compared with ECOG PS at 1 month $(P<.01)$, 3 months $(P<.01)$, and 6 months $(P<.01) .{ }^{109}$

Another prognostic score using decision tree analysis was published by Brims and colleagues ${ }^{110}$ who developed and validated a simple, clinically relevant model to discriminate patients at high and lower risk of death using routinely available variables from the time of diagnosis. The strongest predictive variable was the presence of weight loss. The group with the best survival at 18 months $(86.7 \%$ alive, median survival 34.0 months, termed risk group 1) had no weight loss, an $\mathrm{Hb}$ level of $>153 \mathrm{~g} / \mathrm{L}$, and a serum albumin level of $>43 \mathrm{~g} / \mathrm{L}$. The group with the worst survival $(0 \%$ alive, median survival 7.5 months, termed risk group 4d) had weight loss, a PS of 0 or 1 , and sarcomatoid histologic characteristics. ${ }^{110}$

A Japanese group led by Doi $^{111}$ developed a new Novel Prognostic Risk Classification System. ${ }^{111}$ Pathologic subtype, serum LDH, NLR ratio, and total lesion glycolysis (TLG) in ${ }^{18} \mathrm{~F}-\mathrm{FDG}$ PET/CT were independent and significant prognostic factors. Univariate and multivariate analyses revealed that the significant independent predictors of poor survival outcomes were the nonepithelioid histologic type, increased serum $\mathrm{LDH}$, an NLR of $\geq 5.0$, and a TLG of $\geq 525 \mathrm{~g} .{ }^{111}$

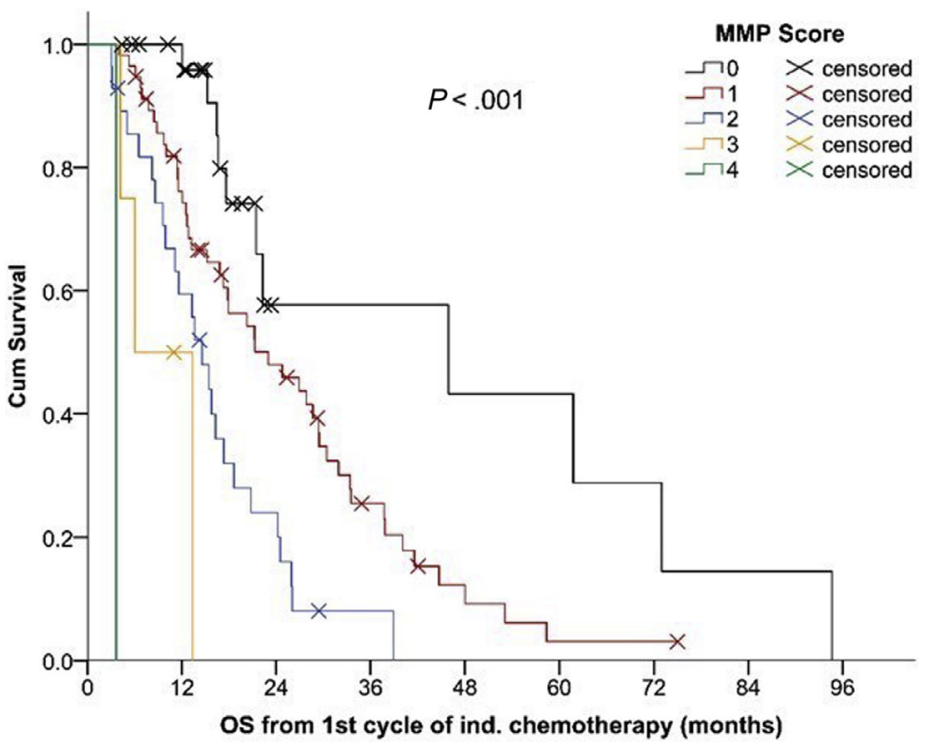

Fig. 3. MMP Score. Kaplan-Meier curve comparing overall survival (OS) according to the multimodality prognostic score (MMPS score) in patients treated with induction chemotherapy followed by MCR. Source: Opitz et al. 2015 unpublished data. 
The problem with most of the scores so far is the lack of validation in independent cohorts and the inclusion of clinical variables being available before surgery to help decision-making for or against surgical resection. ${ }^{112}$ For this purpose, the authors defined in 2012 a multimodality prognostic score (MMPS) to screen patients and define subgroups eligible or especially not eligible for surgery. ${ }^{113}$ The items in the score consisted of tumor volume before chemotherapy $(>500 \mathrm{~mL}$ ), nonepithelioid histologic subtype, CRP greater than $30 \mathrm{mg} / \mathrm{L}$ before chemotherapy, and progressive disease after chemotherapy assessed by mRECIST criteria. The cutoff within this score was at 2 and the specificity of scores 3 and 4 was $100 \%$. The median OS for patients with a score of 0,1 , and 2 was 34,17 , and 12 months, respectively, whereas patients with a score of 3 or 4 had a median OS of 4 months (Fig. 3). The knowledge gained from the MMPS is important for counseling patients because it allows one to reliably identify patients who may have a good chance to benefit from multimodality therapy including surgery and to rule out those in whom aggressive treatment may even cause harm. With a score of 3 or higher, patients are not considered to profit from an MCR in a multimodality therapy approach. ${ }^{113}$ This score was further validated in an independent cohort from Vienna (Fig. 4) treated with the identical multimodality concept, ${ }^{113}$ so that currently the authors are prospectively evaluating the score as an inclusion criterion for clinical trials. ${ }^{112}$ The comparison of MMPS score with EORTC score using ROC curve analysis at 2 years showed that the MMPS demonstrated a better predictive power for OS than the EORTC score. ${ }^{10,113}$

The recommendations from current BTS guidelines for the investigation and management of $\mathrm{MPM}^{42}$ include the following (all grade D). Consider calculating a prognostic score in patients
A
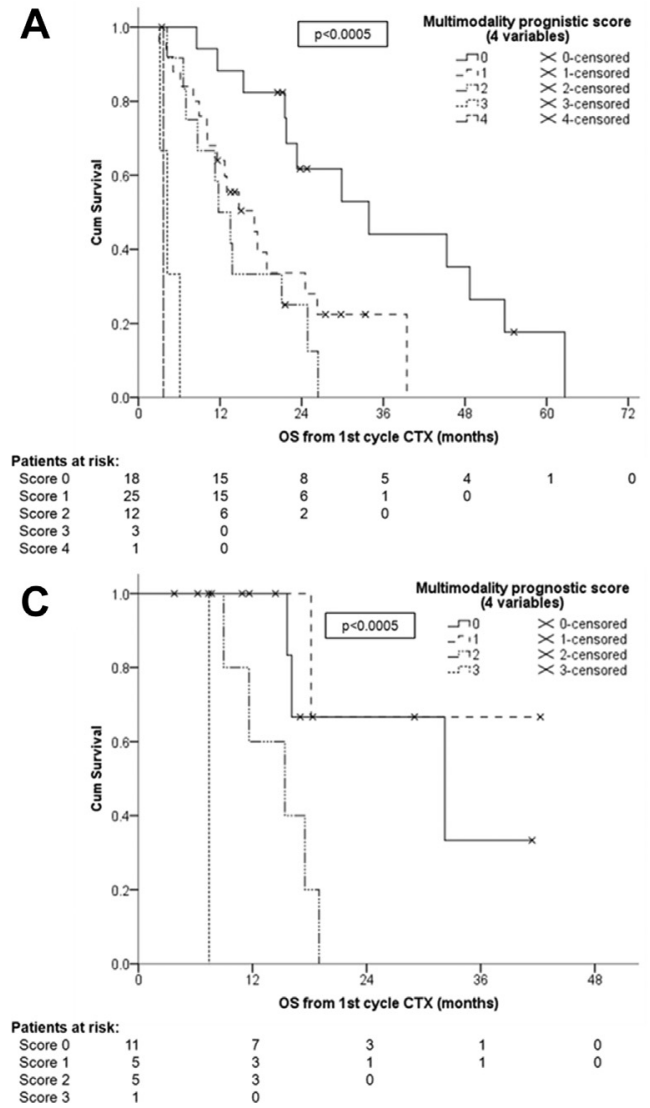

B

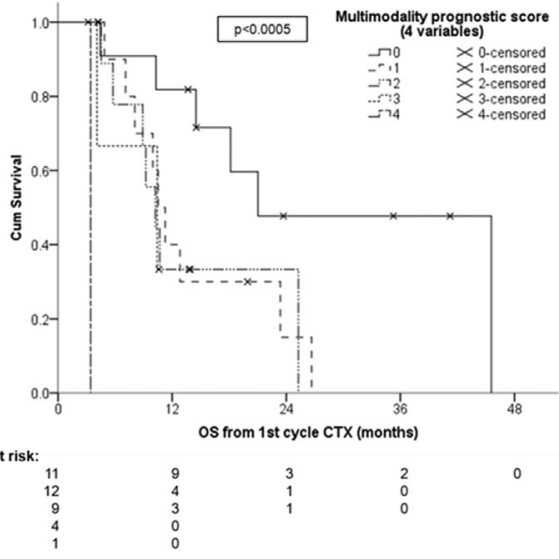

(A)

(B)

(C)

\begin{tabular}{lrrrrrr} 
& $\begin{array}{c}\text { Patients treated with } \\
\text { induction chemotherapy } \\
\text { followed by EPP } \\
\text { (Zurich) }\end{array}$ & $\begin{array}{c}\text { Patients of the } \\
\text { intention to treat } \\
\text { group } \\
\text { (Zurich) }\end{array}$ & $\begin{array}{c}\text { Patients treated with } \\
\text { induction chemotherapy } \\
\text { followed by EPP } \\
\text { (Vienna) }\end{array}$ \\
\hline $\begin{array}{l}\text { number of } \\
\text { patients }\end{array}$ & 59 & \multicolumn{6}{c}{37} & 22 \\
\hline \multicolumn{7}{c}{ Median survival time in months $(95 \%$ Cl) } \\
\hline Score 0 & 34 & $(18-50)$ & 21 & $(6-36)$ & 32 & $(8-56)$ \\
Score 1 & 17 & $(9-25)$ & 11 & $(7-14)$ & - & $(-)$ \\
Score 2 & 12 & $(8-16)$ & 10 & $(8-12)$ & 15 & $(7-24)$ \\
Score 3 & 4 & $(3-6)$ & 10 & $(0-21)$ & 7 & $(-)$ \\
Score 4 & 4 & $(-)$ & 3 & $(-)$ & &
\end{tabular}

Fig. 4. Kaplan-Meier curve of overall survival (OS) in months of the multimodality prognostic score. (A) Patients treated with induction chemotherapy followed by extrapleural pneumonectomy (EPP; Zurich). (B) Patients of the intention-to-treat group (Zurich). (C) Patients treated with induction chemotherapy followed by EPP (Vienna). (From Opitz I, Friess M, Kestenholz P, et al. A new prognostic score supporting treatment allocation for multimodality therapy for malignant pleural mesothelioma: a review of 12 years' experience. J Thorac Oncol. 2015;10(11):1640; with permission.) 
with MPM at diagnosis. Prognostic scores can provide useful survival information for patients and doctors but should not be used in treatment decision-making. When calculating a prognostic score, use one of the following: (a) the EORTC PS; (b) the CALGB score; (c) the mGPS; (d) the LENT score if a pleural effusion is present; (e) the decision tree analysis. The decision tree analysis scoring system is likely to be the most useful in routine clinical practice. ${ }^{42}$ Future investigations needs to extend staging supplemental variables with validation of already available clinical prognostic indices (EORTC and CALGB scores) in combination with laboratory parameters or biomarkers to define the best surgical candidates, as recommended in the ASCO guidelines. ${ }^{6}$

\section{SUMMARY}

Currently there are no generally accepted criteria for patient selection for surgery within a multimodality treatment protocol, but patient selection remains the key parameter for surgical success and low morbidity and mortality. Among clinical, pathologic, and molecular prognostic factors studied in the past, the combination of several factors seems to be the most appropriate tool for decisionmaking, including also institutional experience and availability of therapy as well as factors such as the patient's choice of treatment. Preoperative identification of benefit from surgery comprising a summary of interdisciplinary preoperative workup includes many aspects in this decisionmaking process, including exact diagnosis, staging, validated prognostic scores, multimodal therapy options based on recommendations of the multidisciplinary tumor board, and prospective evaluation of these treatment allocation protocols, when selecting the appropriate therapy for patients in the future. In appropriately selected patients with an acceptable risk profile, surgical resection (extended $P / D, P / D$, or EPP) as part of a multimodality concept should still be offered if performed in high-volume and low-mortality centers, and in any case inclusion in clinical trials or large national or international registries is recommended.

\section{Clinics Care Points}

- Preoperative identification of benefit from surgery is contributed by clinical prognosticators (including age, gender, ECOG PS status, radiologic and surgical staging at baseline and after induction treatment, genetic and molecular factors, laboratory parameters) which, combined with consideration of QoL, are applied often in prognostic scores and allow identification of selected patients for curative approach.

- Future prospective randomized trials must validate all of these approaches to select the best surgical candidates according to approved international guidelines.

\section{DISCLOSURE}

The authors have nothing to disclose.

\section{REFERENCES}

1. Stayner L, Welch LS, Lemen R. The worldwide pandemic of asbestos-related diseases. Annu Rev Public Health 2013;34:205-16.

2. Craighead JE. Epidemiology of mesothelioma and historical background. Recent Results Cancer Res 2011;189:13-25.

3. The Lancet Respiratory M. Pleural mesothelioma: tackling a deadly cancer. Lancet Respir Med 2019;7(2):99

4. Bray F, Ferlay J, Soerjomataram I, et al. Global cancer statistics 2018: GLOBOCAN estimates of incidence and mortality worldwide for 36 cancers in 185 countries. CA Cancer J Clin 2018;68(6): 394-424.

5. Bibby AC, Maskell NA. Current treatments and trials in malignant pleural mesothelioma. Clin Respir J 2018;12(7):2161-9.

6. Kindler HL, Ismaila N, Armato SG 3rd, et al. Treatment of malignant pleural mesothelioma: American Society of Clinical Oncology clinical practice guideline. J Clin Oncol 2018;36(13):1343-73.

7. Butchart EG, Ashcroft T, Barnsley WC, et al. Pleuropneumonectomy in the management of diffuse malignant mesothelioma of the pleura. Experience with 29 patients. Thorax 1976;31(1):15-24.

8. Scherpereel A, Astoul P, Baas P, et al. Guidelines of the European Respiratory Society and the European Society of Thoracic Surgeons for the management of malignant pleural mesothelioma. Eur Respir J 2010;35(3):479-95.

9. Edwards JG, Martin-Ucar AE, Stewart DJ, et al. Right extrapleural pneumonectomy for malignant mesothelioma via median sternotomy or thoracotomy? Short- and long-term results. Eur J Cardiothorac Surg 2007;31(5):759-64.

10. Curran D, Sahmoud T, Therasse P, et al. Prognostic factors in patients with pleural mesothelioma: the European Organization for Research and Treatment of Cancer experience. J Clin Oncol 1998; 16(1):145-52.

11. Herndon JE, Green MR, Chahinian AP, et al. Factors predictive of survival among 337 patients with mesothelioma treated between 1984 and 
1994 by the Cancer and Leukemia Group B. Chest 1998;113(3):723-31.

12. Edwards JG, Abrams KR, Leverment JN, et al. Prognostic factors for malignant mesothelioma in 142 patients: validation of CALGB and EORTC prognostic scoring systems. Thorax 2000;55(9): 731-5.

13. Gonlugur U, Gonlugur TE. Prognostic factors for 100 patients with malignant pleural mesothelioma. Arch Environ Occup Health 2010;65(2):65-9.

14. Gillezeau CN, van Gerwen M, Ramos J, et al. Biomarkers for malignant pleural mesothelioma: a meta-analysis. Carcinogenesis 2019;40(11): 1320-31.

15. Pinato DJ, MauriFA, Ramakrishnan R, etal. Inflammation-based prognostic indices in malignant pleural mesothelioma. J Thorac Oncol 2012;7(3):587-94.

16. Ghanim B, Hoda MA, Winter MP, et al. Pretreatment serum C-reactive protein levels predict benefit from multimodality treatment including radical surgery in malignant pleural mesothelioma: a retrospective multicenter analysis. Ann Surg 2012;256(2): 357-62.

17. Ghanim B, Hoda MA, Klikovits T, et al. Circulating fibrinogen is a prognostic and predictive biomarker in malignant pleural mesothelioma. $\mathrm{Br} J$ Cancer 2014;110(4):984-90.

18. Fleisher LA, Beckman JA, Brown KA, et al. ACC/ AHA 2007 guidelines on perioperative cardiovascular evaluation and care for noncardiac surgery: a report of the American College of Cardiology/ American Heart Association Task Force on Practice Guidelines (Writing Committee to Revise the 2002 Guidelines on Perioperative Cardiovascular Evaluation for Noncardiac Surgery) developed in collaboration with the American Society of Echocardiography, American Society of Nuclear Cardiology, Heart Rhythm Society, Society of Cardiovascular Anesthesiologists, Society for Cardiovascular Angiography and Interventions, Society for Vascular Medicine and Biology, and Society for Vascular Surgery. J Am Coll Cardiol 2007; 50(17):e159-241.

19. Culver BH, Graham BL, Coates AL, et al. Recommendations for a standardized pulmonary function report. An official American Thoracic Society technical statement. Am J Respir Crit Care Med 2017; 196(11):1463-72.

20. Flores RM, Riedel E, Donington JS, et al. Frequency of use and predictors of cancerdirected surgery in the management of malignant pleural mesothelioma in a community-based (Surveillance, Epidemiology, and End Results [SEER]) population. J Thorac Oncol 2010;5(10): 1649-54.

21. Taioli E, Wolf AS, Camacho-Rivera M, et al. Determinants of survival in malignant pleural mesothelioma: a surveillance, epidemiology, and end results (SEER) study of 14,228 patients. PLoS One 2015;10(12):e0145039.

22. Ettinger DS, Akerley W, Borghaei H, et al. Malignant pleural mesothelioma. J Natl Compr Cancer Netw 2012; 10(1):26-41.

23. Yang CJ, Yan BW, Meyerhoff RR, et al. Impact of age on long-term outcomes of surgery for malignant pleural mesothelioma. Clin Lung Cancer 2016;17(5):419-26.

24. Meyerhoff RR, Yang CF, Speicher PJ, et al. Impact of mesothelioma histologic subtype on outcomes in the Surveillance, Epidemiology, and End Results database. J Surg Res 2015;196(1):23-32.

25. Amin W, Linkov F, Landsittel D, et al. Factors influencing malignant mesothelioma survival: a retrospective review of the National Mesothelioma Virtual Bank cohort [version 2; peer review: 2 approved, 1 approved with reservations]. F1000Res 2018;7:1184.

26. Bovolato P, Casadio C, Bille A, et al. Does surgery improve survival of patients with malignant pleural mesothelioma?: a multicenter retrospective analysis of 1365 consecutive patients. J Thorac Oncol 2014;9(3):390-6.

27. Zhuo M, Zheng Q, Chi Y, et al. Survival analysis via nomogram of surgical patients with malignant pleural mesothelioma in the Surveillance, Epidemiology, and End Results database. Thorac Cancer 2019;10(5):1193-202.

28. Taioli E, Wolf AS, Camacho-Rivera M, et al. Women with malignant pleural mesothelioma have a threefold better survival rate than men. Ann Thorac Surg 2014;98(3):1020-4.

29. Linton A, Pavlakis N, O'Connell R, et al. Factors associated with survival in a large series of patients with malignant pleural mesothelioma in New South Wales. Br J Cancer 2014;111(9):1860-9.

30. Pinton G, Brunelli E, Murer B, et al. Estrogen receptor-beta affects the prognosis of human malignant mesothelioma. Cancer Res 2009;69(11): 4598-604.

31. Van Gerwen M, Alpert N, Wolf A, et al. Prognostic factors of survival in patients with malignant pleural mesothelioma: an analysis of the National Cancer Database. Carcinogenesis 2019;40(4): 529-36.

32. van Meerbeeck JP, Gaafar R, Manegold C, et al. Randomized Phase III Study of Cisplatin With or Without Raltitrexed in Patients With Malignant Pleural Mesothelioma: An Intergroup Study of the European Organisation for Research and Treatment of Cancer Lung Cancer Group and the National Cancer Institute of Canada. J Clin Oncol 2005;23(28):6881-9.

33. Ceresoli GL, Grosso F, Zucali PA, et al. Prognostic factors in elderly patients with malignant pleural 
mesothelioma: results of a multicenter survey. $\mathrm{Br} \mathrm{J}$ Cancer 2014;111(2):220-6.

34. Chen N, Liu S, Huang L, et al. Prognostic significance of neutrophil-to-lymphocyte ratio in patients with malignant pleural mesothelioma: a meta-analysis. Oncotarget 2017;8(34):57460-9.

35. Bibby AC, Dorn P, Psallidas I, et al. ERS/EACTS statement on the management of malignant pleural effusions. Eur Respir J 2018;52(1):1800349.

36. Ghanim B, Rosenmayr A, Stockhammer P, et al. Tumour cell PD-L1 expression is prognostic in patients with malignant pleural effusion: the impact of C-reactive protein and immune-checkpoint inhibition. Sci Rep 2020;10(1):5784.

37. Chen ZJ, Gaudino G, Pass HI, et al. Diagnostic and prognostic biomarkers for malignant mesothelioma: an update. Transl Lung Cancer Res 2017; 6(3):259-69.

38. Sun HH, Vaynblat A, Pass HI. Diagnosis and prognosis - review of biomarkers for mesothelioma. Ann Transl Med 2017:5(11):244.

39. Wu T, Zhang W, Yang G, et al. HMGB1 overexpression as a prognostic factor for survival in cancer: a meta-analysis and systematic review. Oncotarget 2016;7(31):50417-27.

40. Tabata C, Shibata E, Tabata R, et al. Serum HMGB1 as a prognostic marker for malignant pleural mesothelioma. BMC Cancer 2013;13:205.

41. Yamagishi T, Fujimoto $N$, Nishi $H$, et al. Prognostic significance of the lymphocyte-to-monocyte ratio in patients with malignant pleural mesothelioma. Lung Cancer 2015;90(1):111-7.

42. Woolhouse I, Bishop L, Darlison L, et al. British Thoracic Society Guideline for the investigation and management of malignant pleural mesothelioma. Thorax 2018;73(Suppl 1):i1-30.

43. Frauenfelder T, Kestenholz P, Hunziker R, et al. Use of computed tomography and positron emission tomography/computed tomography for staging of local extent in patients with malignant pleural mesothelioma. J Comput Assist Tomogr 2015;39(2): 160-5.

44. Martini K, Meier A, Opitz I, et al. Diagnostic accuracy of sequential co-registered $\mathrm{PET}+\mathrm{MR}$ in comparison to $\mathrm{PET} / \mathrm{CT}$ in local thoracic staging of malignant pleural mesothelioma. Lung Cancer 2016:94:40-5.

45. Wang ZJ, Reddy GP, Gotway MB, et al. Malignant pleural mesothelioma: evaluation with CT, MR imaging, and PET. Radiographics 2004;24(1): 105-19.

46. Rusch VW, Venkatraman ES. Important prognostic factors in patients with malignant pleural mesothelioma, managed surgically. Ann Thorac Surg 1999; 68(5):1799-804.

47. Frauenfelder T, Tutic M, Weder W, et al. Volumetry: an alternative to assess therapy response for malignant pleural mesothelioma? Eur Respir J 2011;38(1):162-8.

48. Armato SG 3rd, Blyth KG, Keating JJ, et al. Imaging in pleural mesothelioma: A review of the 13th international conference of the international mesothelioma interest group. Lung Cancer 2016; 101:48-58.

49. Lee HY, Hyun SH, Lee KS, et al. Volume-based parameter of 18)F-FDG PET/CT in malignant pleural mesothelioma: prediction of therapeutic response and prognostic implications. Ann Surg Oncol 2010;17(10):2787-94.

50. Rusch VW, Chansky K, Kindler HL, et al. The IASLC Mesothelioma Staging Project: proposals for the M descriptors and for revision of the TNM stage groupings in the forthcoming (eighth) edition of the TNM Classification for Mesothelioma. J Thorac Oncol 2016;11(12):2112-9.

51. Nowak AK, Chansky K, Rice DC, et al. The IASLC mesothelioma staging project: proposals for revisions of the $T$ descriptors in the forthcoming eighth edition of the TNM classification for pleural mesothelioma. J Thorac Oncol 2016;11(12): 2089-99.

52. Sugarbaker DJ, Flores RM, Jaklitsch MT, et al. Resection margins, extrapleural nodal status, and cell type determine postoperative long-term survival in trimodality therapy of malignant pleural mesothelioma: results in 183 patients. J Thorac Cardiovasc Surg 1999;117(1):54-63 [discussion: 63-65].

53. Sugarbaker DJ, Strauss GM, Lynch TJ, et al. Node status has prognostic significance in the multimodality therapy of diffuse, malignant mesothelioma. J Clin Oncol 1993;11(6):1172-8.

54. Scherpereel A, Astoul P, Baas P, et al. [Guidelines of the European Respiratory Society and the European Society of Thoracic Surgeons for the management of malignant pleural mesothelioma]. Zhongguo Fei Ai Za Zhi 2010;13(10):C23-45.

55. Rice D, Chansky K, Nowak A, et al. The IASLC mesothelioma staging project: proposals for revisions of the $\mathrm{N}$ descriptors in the forthcoming eighth edition of the TNM classification for pleural mesothelioma. J Thorac Oncol 2016;11(12):2100-11.

56. Verma V, Wegner RE, Stahl JM, et al. Impact of detecting occult pathologic nodal disease during resection for malignant pleural mesothelioma. Clin Lung Cancer 2020;21(4):e274-85.

57. Sinha S, Swift AJ, Kamil MA, et al. The role of imaging in malignant pleural mesothelioma: an update after the 2018 BTS guidelines. Clin Radiol 2020; 75(6):423-32.

58. van Klaveren RJ, Aerts JG, de Bruin H, et al. Inadequacy of the RECIST criteria for response evaluation in patients with malignant pleural mesothelioma. Lung Cancer 2004;43(1):63-9. 
59. Byrne MJ, Nowak AK. Modified RECIST criteria for assessment of response in malignant pleural mesothelioma. Ann Oncol 2004;15(2):257-60.

60. Bonomi M, De Filippis C, Lopci E, et al. Clinical staging of malignant pleural mesothelioma: current perspectives. Lung Cancer 2017;8:127-39.

61. Armato SG 3rd, Oxnard GR, MacMahon H, et al. Measurement of mesothelioma on thoracic CT scans: a comparison of manual and computerassisted techniques. Med Phys 2004;31(5): 1105-15.

62. Kindler HL, Ismaila N, Hassan R. Treatment of malignant pleural mesothelioma: American Society of Clinical Oncology clinical practice guideline summary. J Oncol Pract 2018;14(4):256-64.

63. Krug LM, Pass HI, Rusch VW, et al. Multicenter phase II trial of neoadjuvant pemetrexed plus cisplatin followed by extrapleural pneumonectomy and radiation for malignant pleural mesothelioma. J Clin Oncol 2009;27(18):3007-13.

64. Gudmundsson E, Straus CM, Armato SG 3rd. Deep convolutional neural networks for the automated segmentation of malignant pleural mesothelioma on computed tomography scans. J Med Imaging (Bellingham) 2018;5(3):034503.

65. Rusch VW, Gill R, Mitchell A, et al. A multicenter study of volumetric computed tomography for staging malignant pleural mesothelioma. Ann Thorac Surg 2016;102(4):1059-66.

66. Liu F, Zhao B, Krug LM, et al. Assessment of therapy responses and prediction of survival in malignant pleural mesothelioma through computeraided volumetric measurement on computed tomography scans. J Thorac Oncol 2010;5(6): 879-84.

67. Plathow C, Klopp M, Thieke C, et al. Therapy response in malignant pleural mesothelioma-role of MRI using RECIST, modified RECIST and volumetric approaches in comparison with CT. Eur Radiol 2008;18(8):1635-43.

68. Labby ZE, Nowak AK, Dignam JJ, et al. Disease volumes as a marker for patient response in malignant pleural mesothelioma. Ann Oncol 2013;24(4): 999-1005.

69. Armato SG 3rd, Francis RJ, Katz SI, et al. Imaging in pleural mesothelioma: A review of the 14th International Conference of the International Mesothelioma Interest Group. Lung Cancer 2019;130:108-14.

70. Labby ZE, Armato SG 3rd, Dignam JJ, et al. Lung volume measurements as a surrogate marker for patient response in malignant pleural mesothelioma. J Thorac Oncol 2013;8(4):478-86.

71. Pass HI, Temeck BK, Kranda K, et al. Preoperative tumor volume is associated with outcome in malignant pleural mesothelioma. J Thorac Cardiovasc Surg 1998;115(2):310-7 [discussion: 317-318].
72. Sensakovic WF, Armato SG 3rd, Straus C, et al. Computerized segmentation and measurement of malignant pleural mesothelioma. Med Phys 2011; 38(1):238-44.

73. Gill RR, Richards WG, Yeap BY, et al. Epithelial malignant pleural mesothelioma after extrapleural pneumonectomy: stratification of survival with CTderived tumor volume. AJR Am J Roentgenol 2012; 198(2):359-63.

74. Pavic M, Bogowicz M, Wurms X, et al. Influence of inter-observer delineation variability on radiomics stability in different tumor sites. Acta Oncol 2018; 57(8):1070-4.

75. Weber MA, Bock M, Plathow C, et al. Asbestosrelated pleural disease: value of dedicated magnetic resonance imaging techniques. Invest Radiol 2004;39(9):554-64.

76. Burt BM, Lee HS, Raghuram AC, et al. Preoperative prediction of unresectability in malignant pleural mesothelioma. J Thorac Cardiovasc Surg 2019; 159(6):2512-20.e1.

77. Armato SG 3rd, Labby ZE, Coolen J, et al. Imaging in pleural mesothelioma: a review of the 11th International Conference of the International Mesothelioma Interest Group. Lung Cancer 2013;82(2): 190-6.

78. Lim JH, Choi JY, Im Y, et al. Prognostic value of SUVmax on 18F-fluorodeoxyglucose PET/CT scan in patients with malignant pleural mesothelioma. PLoS One 2020;15(2):e0229299.

79. Schwartz RM, Lieberman-Cribbin W, Wolf A, et al. Systematic review of quality of life following pleurectomy decortication and extrapleural pneumonectomy for malignant pleural mesothelioma. BMC cancer 2018;18(1):1188.

80. Rena O, Casadio C. Extrapleural pneumonectomy for early stage malignant pleural mesothelioma: a harmful procedure. Lung Cancer 2012;77(1): 151-5.

81. Ploenes T, Osei-Agyemang T, Krohn A, et al. Changes in lung function after surgery for mesothelioma. Asian Cardiovasc Thorac Ann 2013; 21(1):48-55.

82. Nakas A, Waller D. Predictors of long-term survival following radical surgery for malignant pleural mesothelioma. Eur J Cardiothorac Surg 2014;46(3): 380-5 [discussion: 385].

83. Galateau-Salle F, Churg A, Roggli V, et al. The 2015 World Health Organization classification of tumors of the pleura: advances since the 2004 classification. J Thorac Oncol 2016;11(2): 142-54.

84. Woodard GA, Jablons DM. Surgery for pleural mesothelioma, when it is indicated and why: arguments against surgery for malignant pleural mesothelioma. Transl Lung Cancer Res 2020; 9(Suppl 1):S86-91. 
85. Travis WD, Brambilla E, Burke AP, et al. Introduction to The 2015 World Health Organization classification of tumors of the lung, pleura, thymus, and heart. J Thorac Oncol 2015;10(9):1240-2.

86. Bilecz A, Stockhammer P, Theegarten D, et al. Comparative analysis of prognostic histopathologic parameters in subtypes of epithelioid pleural mesothelioma. Histopathology 2020;77(1):55-66.

87. Musk AW, Olsen N, Alfonso H, et al. Predicting survival in malignant mesothelioma. Eur Respir J 2011; 38(6): 1420-4.

88. Saddoughi SA, Abdelsattar ZM, Blackmon SH. National trends in the epidemiology of malignant pleural mesothelioma: a national cancer data base study. Ann Thorac Surg 2018;105(2):432-7.

89. Nakas A, Trousse DS, Martin-Ucar AE, et al. Open lung-sparing surgery for malignant pleural mesothelioma: the benefits of a radical approach within multimodality therapy. Eur J Cardiothorac Surg 2008;34(4):886-91.

90. Neragi-Miandoab S, Richards WG, Sugarbaker DJ. Morbidity, mortality, mean survival, and the impact of histology on survival after pleurectomy in 64 patients with malignant pleural mesothelioma. Int J Surg 2008;6(4):293-7.

91. Jean D, Daubriac J, Le Pimpec-Barthes F, et al. Molecular changes in mesothelioma with an impact on prognosis and treatment. Arch Pathol Lab Med 2012;136(3):277-93.

92. Baumann F, Flores E, Napolitano A, et al. Mesothelioma patients with germline BAP1 mutations have 7-fold improved long-term survival. Carcinogenesis 2015;36(1):76-81.

93. McGregor SM, Dunning R, Hyjek E, et al. BAP1 facilitates diagnostic objectivity, classification, and prognostication in malignant pleural mesothelioma. Hum Pathol 2015;46(11):1670-8.

94. Bartel DP. MicroRNAs: Genomics, biogenesis, mechanism, and function. Cell 2004;116(2):281-97.

95. Ambros $V$. The functions of animal microRNAs. Nature 2004;431(7006):350-5.

96. Kirschner MB, Cheng YY, Armstrong NJ, et al. MiRscore: a novel 6-microRNA signature that predicts survival outcomes in patients with malignant pleural mesothelioma. Mol Oncol 2015;9(3):715-26.

97. Opitz I, Soltermann A, Abaecherli M, et al. PTEN expression is a strong predictor of survival in mesothelioma patients. Eur J Cardiothorac Surg 2008; 33(3):502-6.

98. Bitanihirwe BK, Meerang M, Friess $M$, et al. PI3K/ mTOR signaling in mesothelioma patients treated with induction chemotherapy followed by extrapleural pneumonectomy. J Thorac Oncol 2014;9(2):239-47.

99. Schramm A, Opitz I, Thies S, et al. Prognostic significance of epithelial-mesenchymal transition in malignant pleural mesothelioma. Eur J Cardiothorac Surg 2010;37(3):566-72.
100. Kuroda A, Matsumoto S, Fukuda A, et al. The mTOR signaling pathway is associated with the prognosis of malignant pleural mesothelioma after multimodality therapy. Anticancer Res 2019; 39(11):6241-7.

101. Otterstrom C, Soltermann A, Opitz I, etal. CD74: a new prognostic factor for patients with malignant pleural mesothelioma. Br J Cancer 2014;110(8):2040-6.

102. Thies S, Friess M, Frischknecht L, et al. Expression of the stem cell factor nestin in malignant pleural mesothelioma is associated with poor prognosis. PLoS One 2015;10(9):e0139312.

103. Meerang M, Berard K, Friess M, et al. Low Merlin expression and high Survivin labeling index are indicators for poor prognosis in patients with malignant pleural mesothelioma. Mol Oncol 2016;10(8): 1255-65.

104. Nguyen BH, Montgomery R, Fadia M, et al. PD-L1 expression associated with worse survival outcome in malignant pleural mesothelioma. Asia Pac J Clin Oncol 2018;14(1):69-73.

105. Sandri A, Guerrera F, Roffinella M, et al. Validation of EORTC and CALGB prognostic models in surgical patients submitted to diagnostic, palliative or curative surgery for malignant pleural mesothelioma. J Thorac Dis 2016;8(8):2121-7.

106. Leuzzi G, Rea F, Spaggiari L, et al. Prognostic score of long-term survival after surgery for malignant pleural mesothelioma: a multicenter analysis. Ann Thorac Surg 2015;100(3):890-7.

107. Rusch VW, Giroux D, Kennedy C, et al. Initial analysis of the international association for the study of lung cancer mesothelioma database. J Thorac Oncol 2012;7(11):1631-9.

108. Pass HI, Giroux D, Kennedy C, et al. Supplementary prognostic variables for pleural mesothelioma: a report from the IASLC staging committee. J Thorac Oncol 2014;9(6):856-64.

109. Clive AO, Kahan BC, Hooper CE, et al. Predicting survival in malignant pleural effusion: development and validation of the LENT prognostic score. Tho$\operatorname{rax} 2014 ; 69(12): 1098-104$.

110. Brims FJ, Meniawy TM, Duffus I, et al. A novel clinical prediction model for prognosis in malignant pleural mesothelioma using decision tree analysis. J Thorac Oncol 2016;11(4):573-82.

111. Doi H, Kuribayashi K, Kitajima K, et al. Development of a novel prognostic risk classification system for malignant pleural mesothelioma. Clin Lung Cancer 2020;21(1):66-74.e2.

112. Opitz I, Weder W. Pleural mesothelioma: is the surgeon still there? Ann Oncol 2018;29(8):1710-7.

113. Opitz I, Friess M, Kestenholz P, et al. A new prognostic score supporting treatment allocation for multimodality therapy for malignant pleural mesothelioma: a review of 12 years' experience. J Thorac Oncol 2015;10(11):1634-41. 